\title{
Evaluation of Electronic Ambulatory Care Data for Use in the Influenza-like Illness Surveillance Network (ILINet)
}

\author{
Kathleen Stigi*1, Atar Baer ${ }^{2}$ and Kathy Lofy ${ }^{1}$ \\ ${ }^{1}$ Communicable Disease Epidemiology, Washington State Department of Health, Shoreline, WA, USA; ${ }^{2}$ Public Health Seattle King \\ County, Seattle, WA, USA
}

\section{Objective}

To determine if a syndromic influenza-like illness (ILI) definition previously validated for emergency department (ED) data accurately identified ILI visits in electronic ambulatory care data.

\section{Introduction}

During summer 2012, Washington State Department of Health (WA DOH) surveyed ILINet providers and found that more than half either utilize their electronic medical record system (EMRS) to gather and report weekly ILINet data, or intend to implement queries to do so in the future. There are a variety of EMRS being used state-wide, and providers that currently utilize these systems to report ILINet data apply a wide range of methods to query their data. There exists great interest in the evaluation of ambulatory care data within the context of Meaningful Use and little research is published in this area. WA DOH sought to evaluate electronic data from WA outpatient clinic networks in order to determine if a syndromic ILI definition previously validated for emergency department (ED) data accurately identified ILI visits in electronic ambulatory care data.

\section{Methods}

Public Health Seattle King County (PHSKC) receives electronic health data from the University of Washington Physicians Network (UWPN), comprised of ten outpatient clinics, on an automated basis. Data are sent daily for all outpatient visits that occurred the previous day and include clinic name, visit date and time, patient age, sex, zip code, chief complaint and diagnoses, and both a visit and patient key. Outpatient data from August 2007 through August 2012 were queried for ILI visits using the syndromic category for ILI previously validated for ED syndromic surveillance data: (1) ICD codes for influenza or mention of "flu" in chief complaint or diagnosis, or (2) a chief complaint or diagnosis of fever plus cough, or (3) a chief complaint or diagnosis of fever plus sore throat.

Using this definition, we assessed the correlation between the proportion of visits for ILI in the UWPN data and number and percentage of positive influenza laboratory tests reported by the University of Washington (UW) Virology Laboratory. We plan to apply this methodology to evaluate outpatient data from an additional clinic network, with statewide locations, and present these findings.

\section{Results}

The median number of weekly visits captured in the data was 6,622 . Three clinics were excluded from further analyses due to insufficient data, leaving seven clinics remaining in the dataset (median number of weekly visits: 6,167). Overall, the proportion of ILI visits in the UWPN data strongly correlated with the number and percentage of positive influenza tests reported by the UW Laboratory during August 2007 through August 2012 (correlation coefficients
0.85 and 0.77 , respectively). The correlation between proportion of ILI visits and number positive influenza tests among individual clinics ranged from $0.62-0.83$. Overall, the proportion of ILI visits among the age category 5 to 24 years most strongly correlated with number positive influenza tests (correlation coefficient: 0.86 ).

\section{Conclusions}

During August 2007 through August 2012, the percentage of ILI visits detected in UWPN data using a previously validated definition for ILI in ED syndromic surveillance data strongly correlated with influenza activity in the community. Based on these findings, data from the UWPN network will be incorporated into ILINet during the 2012-2013 Influenza season. Findings from our analysis support the validity of using syndromic ambulatory data for ILI surveillance. Furthermore, we plan to use these results to formulate guidance for ILINet providers who want to utilize EMRS for weekly ILINet reporting.

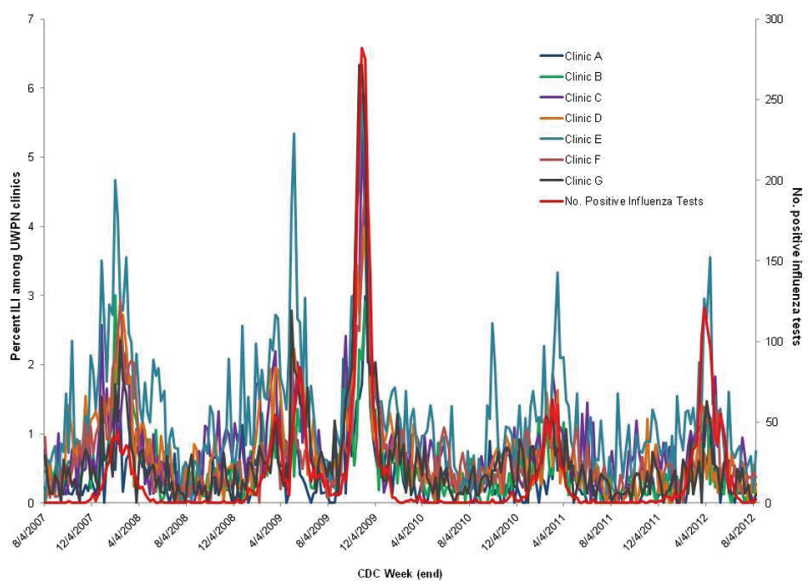

Proportion of ILI visits within electronic clinic network data and number positive influenza tests, August 2007 - August 2012, Washington State

\section{Keywords}

Influenza; ILINet; Influenza-like Illness (ILI); Electronic Medical Records (EMR); Ambulatory data

\section{Acknowledgments}

University of Washington Physicians Network, University of Washington Virology Laboratory, WA State ILINet providers, Natasha Close (WA $\mathrm{DOH})$

\author{
*Kathleen Stigi \\ E-mail: Katie.Stigi@doh.wa.gov
}

